\title{
ON THE COMMUTANT OF A COMPLETE BOOLEAN ALGEBRA OF PROJECTIONS
}

\author{
H. R. DOWSON ${ }^{1}$
}

1. Bade [1], [2] has developed a multiplicity theory for a complete Boolean algebra (B.A.) of projections on a Banach space. The purpose of this paper is to introduce three properties which are shown to be equivalent to uniform multiplicity one for a complete B.A. of projections on a Hilbert space. Examples of Dieudonné show that this result does not hold in general in a Banach space.

2. For a complete discussion of Bade's multiplicity theory the reader is referred to [1] and [2]. However we recall the principal definitions and properties.

A B.A. $\widetilde{B}$ of projections on a Banach space $X$ will be called complete if and only if for every family $\left\{E_{\alpha}\right\} \subseteq \widetilde{B}$ the projections $\vee E_{\alpha}$ and $\Lambda E_{\alpha}$ exist in $\widetilde{B}$ and

$$
\left(\vee E_{\alpha}\right) X=\operatorname{clm}\left\{E_{\alpha} X\right\}, \quad\left(\Lambda E_{\alpha}\right) X=\bigcap\left\{E_{\alpha} X\right\} .
$$

For such a B.A. $\tilde{B}$ of projections, corresponding to each vector $x$ in $X$ there is a carrier projection $C(x)$ in $\widetilde{B}$ defined by

$$
C(x)=\wedge\{E \in \widetilde{B}: E x=x\} .
$$

A B.A. of projections is called countably decomposable if and only if any pairwise disjoint $(E F=0)$ subfamily is at most countable. The restriction of a complete B.A. $\widetilde{B}$ of projections to the range of a carrier projection in $\widetilde{B}$ is both complete and countably decomposable. The cyclic subspace $M(x)$ spanned by a vector $x$ is defined by

$$
M(x)=\operatorname{clm}\{E x: E \in \widetilde{B}\} .
$$

Let $\widetilde{B}$ be a complete B.A. of projections on $X$. Then there is a unique multiplicity function $m(\cdot)$ defined on $\tilde{B}$ such that for each carrier projection $E$ in $\widetilde{B}, m(E)$ is the smallest cardinal power of a family $A$ of vectors with

$$
E X=\operatorname{clm}\{M(x): x \in A\} .
$$

If there is $y$ in $X$ such that $X=M(y)$ then $\widetilde{B}$ is said to have simple spectrum. If $\widetilde{B}$ has simple spectrum then clearly $\widetilde{B}$ has uniform mul-

Received by the editors July 31, 1967 .

${ }_{1}^{1}$ Present address: The University, Glasgow, W.2, Scotland. 
tiplicity one. Examples are easily constructed to show that the converse does not hold in general.

The commutant $\widetilde{B}^{\prime}$ of $\widetilde{B}$ is the family of bounded linear operators which commute with (everything in) $\widetilde{B}$. We denote by $A(\widetilde{B})$ the algebra of operators generated by $\widetilde{B}$ in the uniform operator topology. Bade has shown that for any complete B.A. of projections, $A(\tilde{B})$ is precisely the family of operators which leave invariant every closed subspace invariant under $\widetilde{B}[1$, p. 356].

3. We now introduce four properties pertaining to a complete B.A. $\widetilde{B}$ of projections.

I. $\tilde{B}$ has uniform multiplicity one;

II. $\widetilde{B}^{\prime}$ is equal to $A(\widetilde{B})$;

III. there is no nonzero quasinilpotent in $\widetilde{B}^{\prime}$;

IV. $\widetilde{B}^{\prime}$ is commutative.

In order to prove the first theorem we require the following wellknown elementary result [8, Theorem 1, p. 91].

Lemma. Let $\widetilde{B}$ be a complete B.A. of selfadjoint projections on a Hilbert space $H$. Then for each $x$ in $H$, the orthogonal projection onto the closed subspace $M(x)$ commutes with $\tilde{B}$.

Theorem 1. The properties I, II, III and IV are equivalent for a complete B.A. $\tilde{B}$ of selfadjoint projections on a Hilbert space $H$.

Proof of I implies II. Assume I holds. Let $Y$ be a closed subspace of $H$ invariant under $\widetilde{B}$, and let $y \in Y$. There is $x$ in $C(y) H$ with $M(x)=C(y) H$. The restriction $\widetilde{B}_{0}$ of $\widetilde{B}$ to $C(y) H$ is a complete B.A. of projections with simple spectrum. Let $T \in \widetilde{B}^{\prime}$. Then $T C(y)=C(y) T$ and the restriction $T_{0}$ of $T$ to $C(y) H$ is in $\tilde{B}_{0}^{\prime}$. Hence a result of Bade $\left[1\right.$, p. 355] shows $T_{0}$ leaves invariant $M(y) \subseteq C(y) H$. Therefore

$$
T y=T_{0} y \in M(y) \subseteq Y .
$$

Since $Y$ and $y$ are arbitrary it follows that $T \in A(\widetilde{B})$ and so $\widetilde{B}^{\prime} \subseteq A(\widetilde{B})$. Finally $A(\widetilde{B}) \subseteq \widetilde{B}^{\prime}$ and so this part of the proof is complete.

Proof of II implies III. Assume II holds. Let $\Omega$ be the Stone representation space of $\widetilde{B}$. Then $\Omega$ is an extremally disconnected compact Hausdorff space. There is a bicontinuous algebra isomorphism from $A(\widetilde{B})$ onto $C(\Omega)$, the continuous functions on $\Omega$ under the supremum norm $\left[1\right.$, p. 354] . Now $\left\|f^{n}\right\|^{1 / n}=\|f\|, n=1,2,3, \cdots$ for every $f$ in $C(\Omega)$. Hence there is no nonzero quasinilpotent in $A(\widetilde{B})=\widetilde{B}^{\prime}$ and so II implies III.

Proof of III implies I. Assume that $\widetilde{B}$ does not have uniform multiplicity one. Then there is $x$ in $H$ such that $C(x) H \neq M(x)$. Let $y$ 
be a nonzero vector in the orthogonal complement of $M(x)$ in $C(x) H$ and let $P$ be the orthogonal projection from $C(x) H$ onto $M(x)$. By the lemma

$$
P E y=E P y=0, \quad E \in \widetilde{B},
$$

and so $M(y)$ is orthogonal to $M(x) . \widetilde{B}$ may be regarded as a spectral measure defined on $\Sigma_{\Omega}$, the Borel field of its Stone representation space $\Omega$. Define $\mu_{x}(\tau)=\langle E(\tau) x, x\rangle$ and $\mu_{y}(\tau)=\langle E(\tau) y, y\rangle, \tau \in \Sigma_{\Omega}$. We show that $\mu_{y}$ is absolutely continuous $(\ll)$ with respect to $\mu_{x}$. If $\tau \in \Sigma_{\Omega}$, then

$$
\begin{aligned}
\mu_{x}(\tau)=0 & \Rightarrow C(x) E(\tau) x=0 \Rightarrow C(x) E(\Omega \backslash \tau) x=x \\
& \Rightarrow C(x) E(\Omega \backslash \tau)=C(x) \\
& \Rightarrow C(x) E(\tau)=0 \Rightarrow \mu_{y}(\tau)=0 .
\end{aligned}
$$

Since $\mu_{y} \ll \mu_{x}$, it follows from the Radon-Nikodym theorem [6, p. 174] that there is $f$ in $L^{1}\left(\mu_{x}\right)$ such that

$$
\mu_{y}(\tau)=\int_{\tau} f(t) \mu_{x}(d t), \quad \tau \in \Sigma_{\Omega}, \quad f(t) \geqq 0 .
$$

Now $1 /(1+f)$ is measurable and essentially bounded. Hence $1 /(1+f)$ $\in L^{\infty}\left(\mu_{x}\right)$. Define for each $g$ in $L^{2}\left(\mu_{y}\right)$ the function $Q g$ by setting

$$
(Q g)(t)=(f(t) /(1+f(t)))^{1 / 2} g(t) .
$$

From [6, III.10.5, p. 179] we obtain

$$
\begin{aligned}
g \in L^{2}\left(\mu_{y}\right) & \Rightarrow|g|^{2} \in L^{1}\left(\mu_{y}\right) \Rightarrow f|g|^{2} \in L^{1}\left(\mu_{x}\right) \\
& \Rightarrow \frac{f}{1+f} \cdot|g|^{2} \in L^{1}\left(\mu_{x}\right) \\
& \Rightarrow\left(\frac{f}{1+f}\right)^{1 / 2} g \in L^{2}\left(\mu_{x}\right) .
\end{aligned}
$$

Therefore $Q$ is a linear map from $L^{2}\left(\mu_{y}\right)$ into $L^{2}\left(\mu_{x}\right)$. Also $Q$ is bounded, since by $[6$, III.10.5, p. 179]

$$
\begin{aligned}
\|Q g\|^{2} & =\int_{\Omega} \frac{f(t)}{1+f(t)} \cdot|g(t)|^{2} \mu_{x}(d t) \\
& \leqq \int_{\Omega} f(t)|g(t)|^{3} \mu_{x}(d t) \\
& =\int_{\Omega}|g(t)|^{2} \mu_{y}(d t)=\|g\|^{2} .
\end{aligned}
$$


Now $M(x)$ and $M(y)$ are respectively isometrically isomorphic to $L^{2}\left(\mu_{x}\right)$ and $L^{2}\left(\mu_{y}\right)$ [8, Theorem 1, p. 95]. Hence there are unitary operators $U_{x}$ mapping $M(x)$ onto $L^{2}\left(\mu_{x}\right)$ and $U_{y}$ mapping $M(y)$ onto $L^{2}\left(\mu_{y}\right)$. Define a bounded linear operator $N$ on $H$ by

$$
N z=U_{x}^{-1} Q U_{y} z, \quad z \in M(y),
$$

and $N$ is 0 on the orthogonal complement of $M(y)$ in $H$. Clearly $N \neq 0$. Since $M(x)$ and $M(y)$ are orthogonal, $N^{2}=0$. Also by construction $N E=E N, E \in \widetilde{B}$, and so $N$ is a nonzero quasinilpotent operator in $\widetilde{B}^{\prime}$. This shows that III implies I.

Proof OF IV IMPLies I. Suppose that I does not hold. The operator $N$, constructed above under this assumption, does not leave invariant $M(y)$ and so $N$ does not commute with the orthogonal projection $P$ of $H$ onto $M(y)$. Hence by the lemma we have $P \in \widetilde{B}^{\prime}, N \in \widetilde{B}^{\prime}$ and $P N \neq N P$. This shows that IV implies I. Since I implies II and trivially II implies IV the proof of the theorem is complete.

By using the appropriate version of the Mackey-Wermer theorem, we obtain a generalization of Theorem 1. (See for example [5, p. 222].)

Theorem. Let $H_{1}$ be a Hilbert space and let $\widetilde{B}_{1}$ be a bounded B.A. of projections on $H_{1}$ with Stone representation space $\Omega$. Then there exist a Hilbert space $\mathrm{H}_{2}$, a B.A. $\widetilde{B}_{2}$ of selfadjoint projections on $\mathrm{H}_{2}$ with Stone representation space $\Omega$, and a one-to-one bicontinuous linear map $T$ of $\mathrm{H}_{1}$ onto $\mathrm{H}_{2}$ such that

$$
E_{2}(\tau)=T E_{1}(\tau) T^{-1}, \quad \tau \text { open-and-closed in } \Omega .
$$

We observe that it follows readily that if $\widetilde{B}_{1}$ is complete then so is $\widetilde{B}_{2}$. It is also easily verified that the $\operatorname{map} E_{1}(\tau) \rightarrow T E_{1}(\tau) T^{-1}$ and the inverse map preserve the properties I, II, III and IV. Hence we have the following generalization of Theorem 1 .

ThEOREM 2. The properties I, II, III and IV are equivalent for a complete B.A. of projections on a Hilbert space.

4. In conclusion we consider the corresponding problem in a Banach space.

Theorem 3. Let $\widetilde{B}$ be a complete B.A. of projections, with uniform finite multiplicity n, on a Banach space X. Then I implies II, II implies III and III implies IV.

Proof. The proof that I implies II, given in Theorem 1, works in this case too. The proof that II implies III is valid for any complete B.A. of projections, not necessarily of uniform finite multiplicity. 
Assume now that III holds and IV fails. Then there is $x$ in $X$ and $T_{1}, T_{2}$ in $\tilde{B}^{\prime}$ with $T_{1} T_{2} x \neq T_{2} T_{1} x$. Now since there is no nonzero quasinilpotent in $\widetilde{B}^{\prime}$, the B.A. $\tilde{B}_{0}$ obtained by restricting $\widetilde{B}$ to $C(x) X$ has the same property. Since $\widetilde{B}_{0}$ is countably decomposable it follows from $\left[9\right.$, Theorem 7, p. 223] that $\widetilde{B}_{0}^{\prime}$ is commutative. This contradicts the fact that the operators formed by restricting $T_{1}$ and $T_{2}$ to $C(x) X$ are in $\widetilde{B}_{0}^{\prime}$ and fail to commute. Hence III implies IV. (In fact one can deduce similarly from Corollary 8 of [9] that III implies IV for a complete B.A. of projections containing no projection of infinite uniform multiplicity.)

Two examples constructed by Dieudonné show that even for a complete B.A. of projections of uniform finite multiplicity II does not necessarily imply I, and III does not necessarily imply II. In [4] there is an example of a complete B.A. $\widetilde{B}$ of projections with uniform multiplicity two such that $\widetilde{B}^{\prime}=A(\widetilde{B})$. In $[3]$ it is shown that there is a complete B.A. $\widetilde{B}$ of projections with uniform multiplicity two such that $A(\widetilde{B})$ is a proper subalgebra of $\tilde{B}^{\prime}$ and such that there is no nonzero nilpotent in $\tilde{B}^{\prime}$. A result of Foguel shows that in this case there is no nonzero quasinilpotent in $\tilde{B}^{\prime}[\mathbf{7}, \mathrm{p} .687]$. It would seem to be an unsolved problem whether for a complete B.A. of projections of uniform finite multiplicity IV implies III.

\section{REFERENCES}

1. W. G. Bade, On Boolean algebras of projections and algebras of operators, Trans. Amer. Math. Soc. 80 (1955), 345-359.

2. - A multiplicity theory for Boolean algebras of projections on Banach spaces, Trans. Amer. Math. Soc. 92 (1959), 508-530.

3. J. Dieudonné, Sur la bicommutante d'une algèbre d'operateurs, Portugal. Math. 14 (1955), 35-38.

4. - Champs de vecteurs non localement triviaux, Arch. Math. 7 (1956), 6-10.

5. J. Dixmier, Les moyennes invariantes dans les semi-groups et leurs applications, Acta Sci. Math. Szeged 12 (1950), 213-227.

6. N. Dunford and J. T. Schwartz, Linear operators. I, Interscience, New York, 1958.

7. S. R. Foguel, Boolean algebras of projections of finite multiplicity, Pacific J. Math. 9 (1959), 681-693.

8. P. R. Halmos, Introduction to Hilbert space, Chelsea, New York, 1951.

9. L. Tzafriri, On multiplicity theory for Boolean algebras of projections, Israel J. Math. 4 (1966), 217-224.

UNIVERSITY OF ILLINOIS 